\title{
On the dispersion of lithium-sulfur cathode materials effected by electrostatic and stereo-chemical factors of binders
}

\author{
Xiaoheng Hong ${ }^{\mathrm{a}, \mathrm{b}}$, Jun Jin ${ }^{\mathrm{a}}$, Zhaoyin Wen ${ }^{\mathrm{a}, \mathrm{b} *}$, Sanpei Zhang ${ }^{\mathrm{a}}$, Qingsong Wang ${ }^{\mathrm{a}}$, Chen Shen ${ }^{\mathrm{a}}$, Kun Rui ${ }^{\mathrm{a}}$ \\ ${ }^{a}$ CAS Key Laboratory of Materials for Energy Conversion, Shanghai Institute of Ceramics, Chinese Academy of Sciences,
} Shanghai, 200050, P.R. China.

${ }^{b}$ Shanghaitech University, Shanghai, 200031, P.R.China

\begin{abstract}
Sodium carboxymethyl cellulose- styrene butadiene rubber (CMC-SBR), sodium alginate (SA) and LA132 are utilized as the polymer binders for the cathodes of Li-S batteries to study their dispersion mechanism on the cathode materials and the consequent influence on the performance of Li-S batteries. Zeta potential tests, differential scanning calorimetry analysis and calculations of the rotational barriers of the links of the polymer chains by General Atomic and Molecular Electronic Structure System (GAMESS) reveal that higher charge densities and better chain flexibility of the binders promise the dispersion of the downsized cathode materials. LA132 is found to have optimal characteristic for dispersing and stabilizing the cathode materials in aqueous environment. The cycling performance and SEM images of the cathodes demonstrate that cathodes with higher dispersion degree achieve higher discharge capacities. The electrochemical impedance spectroscopy (EIS) results further support that better dispersed cathodes have lower impedance resulting from their well established conducting frameworks.
\end{abstract}

Keywords: lithium-sulfur battery; binder; dispersion mechanism; sulfur cathode; cathode morphology

Corresponding author. Tel: +86-21-52411704, Fax: +86-21-52413903

Email address: zywen@mail.sic.ac.cn

\section{Introduction:}

The continued demand for long-range electric vehicles is impelling the current electrical storage systems beyond the conventional lithium ion batteries. Based on the reversible electrochemical reaction: $\mathrm{S}_{8}+16 \mathrm{Li} \leftrightarrow 8 \mathrm{Li}_{2} \mathrm{~S}[1]$, lithium- sulfur 
battery (Li-S) theoretically offers an energy density of $2600 \mathrm{Wh} \mathrm{kg}^{-1}$ and a specific capacity of $1675 \mathrm{mAh} \mathrm{g}^{-1}$,

demonstrating the potential for being a next-generation energy storage system. Nevertheless, Li-S suffers from capacity decay and self-discharge caused by both polysulfide shuttle effect[2] and the insulating nature of the electrochemical reactive material $\mathrm{S}_{8}$ and $\mathrm{Li}_{2} \mathrm{~S}_{2} / \mathrm{Li}_{2} \mathrm{~S}_{2}$ [3]. Before being scaled for industrial applications, Li-S is still faced with challenges.

To address this, one main strategy is to immobilize sulfur inside downsized conducting host materials e.g. mesoporous carbon[4, 5], carbon nanotube[6, 7], graphene[8, 9], etc. These methods enormously enhance the performance of Li-S battery. However, when it comes to the industrialization of Li-S, the use of hydrophobic materials with high surface area that spontaneously over-aggregate $[10,11]$ still complicates the fabrication of the cathode materials. Severe aggregation of cathode materials would take place when solvent is evaporated. This would lead to undesired pinholes or crackings, deteriorating the cycling performance[12] and causing unreliable quality from batch to batch[13].

To mitigate this, polymer binders play an important role. The basic function of a binder is to strongly interact with both the cathode material and the current collector to enable sufficient electrical contact and structural robustness. More significantly, to overcome the spontaneous over-aggregation, another required function of a binder is to modify the liquidsolid interface and disperse the downsized material homogenously. Polyvinylidene fluoride (PVDF) serves as the most commonly used binder in lithium sulfur batteries for its strong adhesion strength and good electrochemical stability. However, the hydrophobic nature of it brings about the problem of swelling and dissolving in organic electrolyte. This would cause the gradual degradation of the conductive network. Moreover, the solvent for PVDF, N-methyl pyrrolidinone (NMP), is a hazardous organic solvent $[14,15]$ with a boiling point of $230^{\circ} \mathrm{C}$. It requires high energy cost and processing complexity when being dried. In contrast, water base binders are better choices for Li-S application. They do not dissolve in electrolyte owing to the hydrophilic nature, and the solvent water is totally benign. The water based binders in common use are polyethylene oxide (PEO)[16], sodium carboxymethyl cellulose- styrene butadiene rubber (CMC-SBR)[17], sodium alginate (SA)[18], polyvinylpyrrolidone (PVP)[19], carbonyl- $\beta$-cyclodextrin[20], gelatin[21] and LA132[22] (a copolymer of acrylonitrile, acrylamide and acrylic), etc. Among these, SA, CMC-SBR and LA132 are widely applied for dispersing 
and binding different types of sulfur cathode composites [23-27]. They are also of ionic nature originating from the carboxylate groups, which are able to disperse the hydrophobic particles through the electrostatic double-layer repulsion mechanism $[28,29]$. The electrostatic repulsing dispersablity is practically helpful to impart stability and uniformity to the cathode slurry during coating and drying process, which is beneficial to the cycling performance of the cells fabricated.

In this study, we have investigated the electrostatic repulsing effects of these ionic binders on the cathode materials and their consequent impact on the electrochemical performance of the cells. The intensity of electrostatic double layer repulsion were examined by zeta potential tests. The different chain flexibilities of the binders that effects the electrostatic repulsion were studied by determining the glass transition temperature $\left(\mathrm{T}_{\mathrm{g}}\right)$ using differential scanning calorimetry(DSC). The chain flexibilities were also explored by calculation of the rotational barriers of the polymer chains links using General Atomic and Molecular Electronic Structure System (GAMESS). Moreover, Li-S cells with SA, CMC-SBR and LA132 as the binder, respectively, were prepared and tested at controlled conditions in terms of sulfur loading, electrolyte volume, cutoff voltages and cycling rates etc. to manifest how dispersion morphology effects the cell performance. SEM images of the cathodes and the Nyquist plots of the cells are given to verify the discussion.

\section{Experimental}

\subsection{Materials}

The Sulfur-Ketjen Black composite (S-KB) was prepared via heating the mixture of KB and sulfur powder (weight ratio $\mathrm{S}: \mathrm{KB}=4: 1$ ) in a sealed container at $155^{\circ} \mathrm{C}$ for 12 hours. The conductive agent of Super $\mathrm{P}$ carbon black (SP) was used as received. The binders, LA132 (Indigo, Chendu, China), CMC (Mac 350HC, Nippon Paper, Japan), SBR (TRD 102A, JSR, Japan) and SA (Sino pharm Chemical Reagent Co., Ltd, China), were used as received.

\subsection{Cathode preparation and performance test}

The cathode slurry was composed of $80 \mathrm{wt} \%$ of S-KB, $10 \mathrm{wt} \%$ of Super P and $10 \mathrm{wt} \%$ of binders dispersed in water solvent. For CMC-SBR binder, the weight ratio of CMC: SBR is 1:1. All the slurry was prepared by thoroughly mixing the components in deionized water using magnetic stirring for 10 hours. The slurry was blade-coated onto Al foils. After that 
the cathode sheets were hot air dried and further vacuum-dried at $60^{\circ} \mathrm{C}$ for overnight. The obtained cathode sheets were cut into $12 \mathrm{~mm}$ diameter discs with an average sulfur loads of around $0.7-1 \mathrm{mg} \mathrm{cm}^{-2} .2025$ coin-type cells were assembled in an argon-filled glove box (Super1220/ 750/900, MIKROUNA, Shanghai, China) with both $\mathrm{H}_{2} \mathrm{O}$ and $\mathrm{O}_{2}$ contents lower than 0.1 ppm. The electrolyte was composed of $1 \mathrm{~mol} \mathrm{~L}^{-1} \mathrm{LiTFSI}$ and $0.2 \mathrm{~mol} \mathrm{~L}^{-1} \mathrm{LiNO} 3$ in a mixture of 1, 3-dioxolane (DOL) and 1, 2-dimethoxyethane (DME) (1:1 volume ratio). Metallic lithium foils were used as the anodes and polymer membranes (SK, Korea) served as the separators. The volume of electrolyte for each cell was controlled at $40 \mu \mathrm{L}$. The cells were tested at room temperature (approx. $\left.25^{\circ} \mathrm{C}\right)$ under galvanostatic condition at $0.2 \mathrm{C}\left(335 \mathrm{~mA} \mathrm{~g}^{-1}\right)$. The cut off voltage was $1.8 \mathrm{~V}$ to 2.8 V vs. Li/Li+. The charge-discharge test instrument was CT2001A from LAND Electronic Co., Ltd, China.

\subsection{Electrochemical impedance spectroscopy}

The electrochemical impedance spectroscopies (EIS) of the cells were measured at the open circuit voltage on Autolab testing platform (Metrohm, Switzerland). The frequency range applied was from $10^{6} \mathrm{~Hz}$ to $0.1 \mathrm{~Hz}$ with an amplitude of $0.01 \mathrm{~V}$.

\subsection{Zeta potential test}

$\mathrm{S}-\mathrm{KB}$ were dispersed in aqueous solutions of CMC, SA and LA132 by ultrasonic treatment to give homogenous colloids. A series of colloids for each binder were prepared. The concentrations of S-KB were controlled at $4 \mu \mathrm{gL}^{-1}$ and the concentrations of the binder were $4,8,16$ and $48 \mu \mathrm{g} \mathrm{mL}^{-1}$, respectively. The zeta potential of the particles were tested on Malvern Zetasizer Nano-ZS90.

\subsection{Differential scanning calorimetry}

DSC analysis was carried out on Mettler Toledo DSC 822. Before DSC analysis, the samples were vacuum dried at $60^{\circ} \mathrm{C}$ for over night to dehumidify.7.00mg of LA132, 7.00mg of CMC and 7.20mg of SA were tested. The samples were heated from $25^{\circ} \mathrm{C}$ to $180^{\circ} \mathrm{C}$ at $5^{\circ} \mathrm{C} \mathrm{min}^{-1}$.

\subsection{SEM study}


The morphology of the cathode of each binder, before and after the cycling tests, were observed using a scanning electron microscopy (SEM, S3400N, Hitachi). Before the SEM observation, the cathode discs were gently rinsed in DME in the glove box to remove the LiTFSI remaining.

\subsection{Peel test}

$180^{\circ}$ peel tests were performed on a universal testing instrument (Instron-5592, Massachusetts, U.S.A.). The cathode film was sandwiched between a $3 \mathrm{M}$ scotch tape and the $\mathrm{Al}$ foil. The peeling rate employed was $50 \mathrm{~mm} \mathrm{~min}^{-1}$.

\section{Results \& Discussion}

Since the dried cathode retains the morphology of the wet state, the cathode morphology is dependent on the dispersion degree of the cathode slurry. In this way, the dispersability of the binder effects the electrochemical performance of a Li-S cell. The binders studied here, CMC-SBR, SA and LA132, are all of ionic nature stemming from the carboxyl groups on the chains. The chain segments adsorb onto the particles to lower the surface energy, and charge the particles negatively to form electrical repulsion among the particles. The charge developed on the particles that facilitates the dispersion was characterized by zeta potential[30]. Figure 1 gives the zeta potential of the S-KB particles in different binder solutions at incremental concentrations. Here the case for SBR of CMC-SBR binder system was not investigated because of its nonionic character.

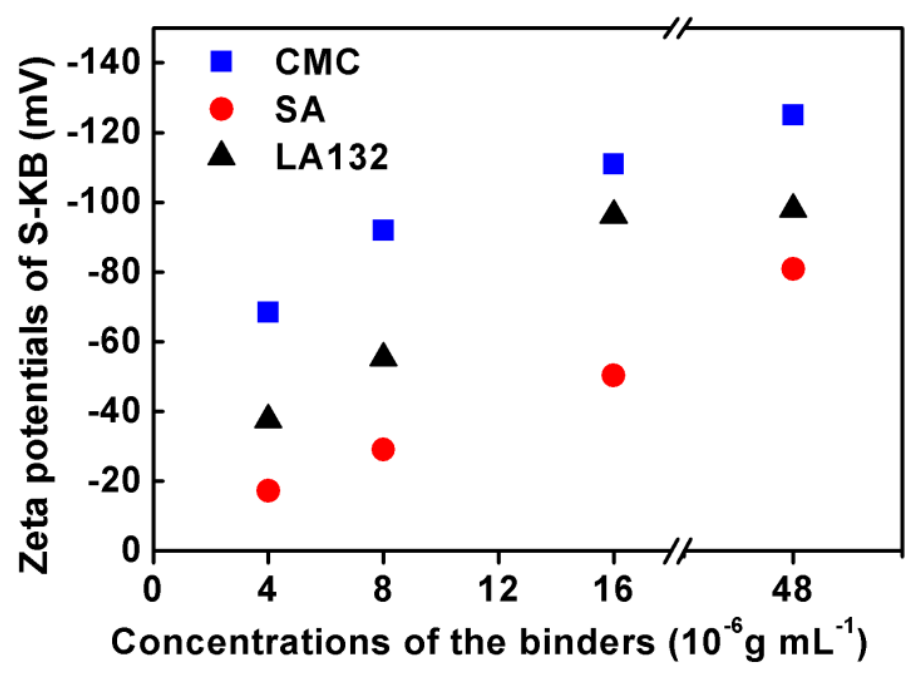

Figure 1. The zeta potentials of the S-KB dispersed in incremental concentration of each binders. 
The zeta potential results indicate that the particles are all negatively charged by the - $\mathrm{COO}^{-}$groups. With the increased concentrations of the binders, there is corresponding increase in the charge accumulation. The charges on the particles form electrical double layers at the solid-liquid interface[31]. As the double layers are of the same sign on all the particles, the interaction is repulsive which prevents the aggregation. CMC solution shows the best ability of charging the particles, while LA132 comes after and SA shows the poorest performance. The gaps probably root in the difference of their chemical structures.
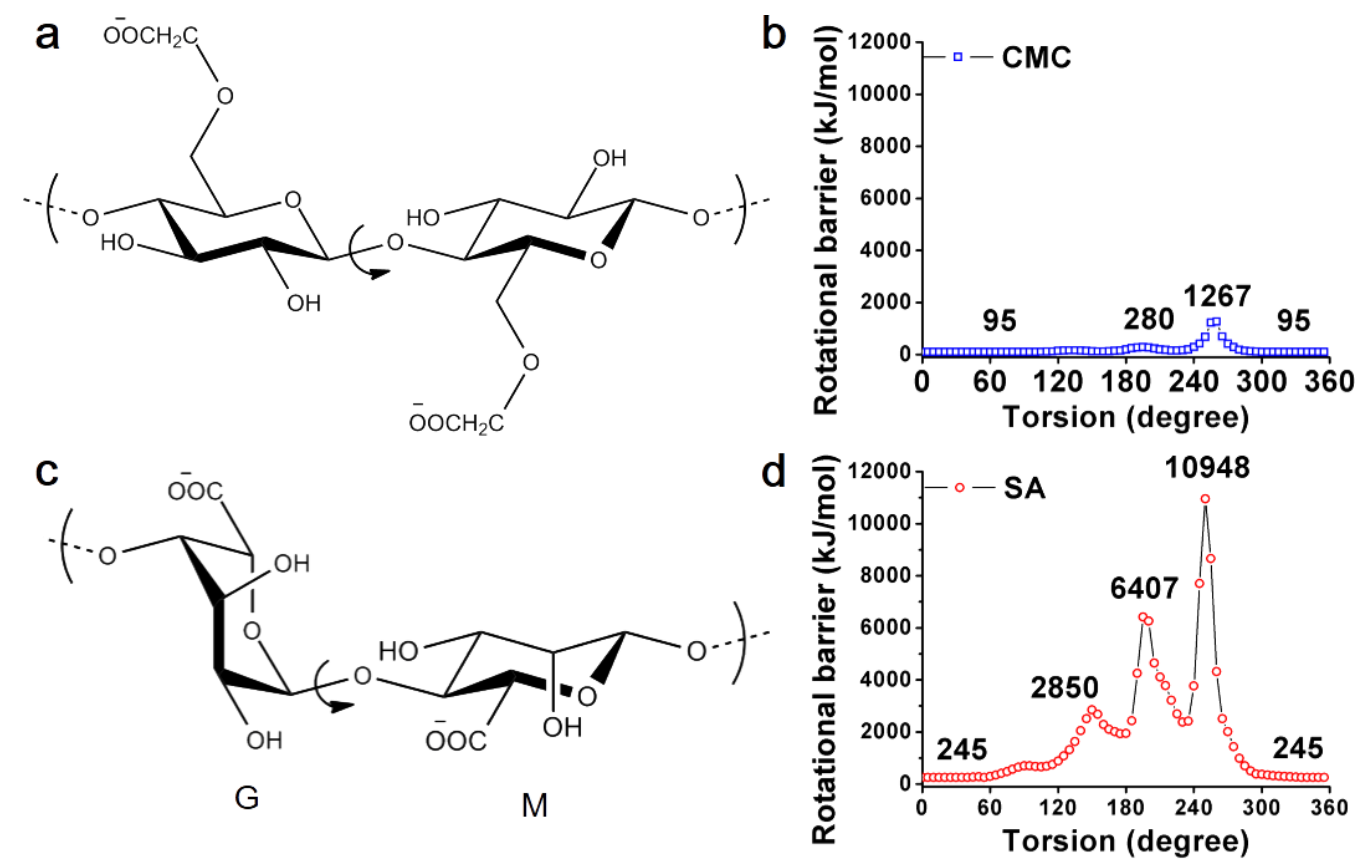

Figure 2. (a) Chemical structure of $\mathrm{CMC}, \mathrm{DS}=1$; (b) the rotational barrier of the equatorial linkage of $\mathrm{CMC}$. The calculation is based on the rotation about the equatorial bond marked with the arrow in figure 2a; (c) chemical structure of SA; (d) the rotational barrier of the axial linkage of SA. The calculation is based on the rotation about the axial bond marked with the arrow in figure $2 \mathrm{c}$.

CMC (Figure 2a) is derived from hydrophobic cellulose with varying degrees of carboxymethyl substitution (DS) up to 3 (three $-\mathrm{OH}$ groups ring available for substitution per each ring). The $-\mathrm{CH}_{2} \mathrm{COONa}$ groups render the chains soluble in aqueous environment compared to insoluble cellulose. Meanwhile, the main chains of cellulose remain hydrophobic[32] which adsorb onto the alike sulfur-carbon particles by hydrophobic interaction. The monomeric units of CMC are linked by equatorial oxygen bonds (labeled with an arrow in figure 2a) which allows smaller rotational barrier for conformation 
changes of the chain. Hence, the $-\mathrm{COO}^{-}$groups are able to adopt more favorable spatial positions when charging the particles. Compared to CMC, SA (Figure 2c) is a linear copolymer with blocks of (1-4)-linked $\beta$-D-mannuronate (M) and $\alpha$ L-guluronate $(\mathrm{G})$ residues. It could be regarded as a block copolymer comprising of homopolymeric regions of M and $\mathrm{G}$, where alternating structure of MG are interspersed[33]. The G blocks are linked with adjacent blocks by axial bonds as labeled with an arrow in figure 2c, which bring about higher rotational barrier than the equatorial bonds[34]. To further explore the difference between the rotational barriers of CMC and SA, the rotational barrier energies about the equatorial bond of CMC (figure $2 b$ ) and the axial bond of SA (figure 2d) were calculated, respectively, using GAMESS program[35, 36]. For the equatorial bond of $\mathrm{CMC}$, the chain was assumed of two monomeric units where-COOH groups were fully ionized, and the torsion about the equatorial bond was from $0^{\circ}$ to $360^{\circ}$. For the axial bond of SA, the chain was assumed of two monomeric units of a $\mathrm{G}$ and an $\mathrm{M}$ where - $\mathrm{COOH}$ groups were fully ionized as well, and the torsion about the axial bond was also from $0^{\circ}$ to $360^{\circ}$. The gap between the rotational barrier energies of the equatorial bond of CMC and the axial bond of SA is enormous, indicating the much more hindered rotation of the SA chains. Therefore, the chains of SA are much stiffer and allow lower conformational changeability than CMC. With less hindered rotation, when interacting with the particles, CMC chains are flexible to adopt favorable spatial positions to charge the particles more effectively. This accounts for the difference of the zeta potential results of SA and CMC with similar chemical composition.

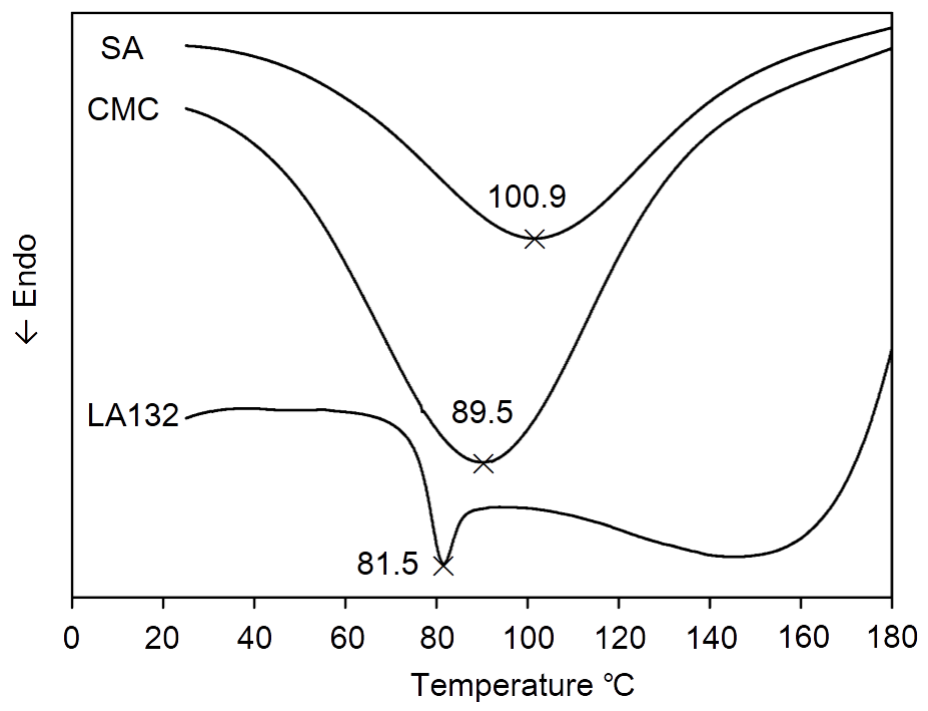

Figure 3. DSC curves of LA132, CMC and SA. The $\mathrm{T}_{\mathrm{g}}$ of each binder is marked with a cross. 
As mentioned, LA132 is a copolymer of acrylonitrile, acrylamide and acrylate where acrylonitrile makes up the basis.

Therefore, compared to the $\mathrm{COO}^{-}$groups of $\mathrm{SA}$ or $\mathrm{CMC}$ which are on every chain units, the $-\mathrm{COO}^{-}$groups of $\mathrm{LA} 132$ only appear on the acrylate units distributed over the chains. This leads to a lower density of -COO- groups on LA132. On the other hand, the backbones of LA132 of vinyl-vinyl structure are of higher flexibility than the ring-ring structure of SA and CMC. Hence, LA132 is more flexible to alter the conformations and charge the particles. To give a comparison of the chain flexibility of the binders, DSC tests were performed to find the glass transition temperatures $\left(\mathrm{T}_{\mathrm{g}}\right)$ of the binders[37]. $\mathrm{T}_{\mathrm{g}}$ is the temperature at which the polymer molecules frozen in the position in the glass state become free to rotate. The most important factor that controls the $\mathrm{T}_{\mathrm{g}}$ of a polymer is chain flexibility[38]. Polymer chains with better flexibility are easier to rotate about the bonds and thus has lower $\mathrm{T}_{\mathrm{g}}$. DSC analysis gives the $\mathrm{T}_{\mathrm{g}}$ of the three binders used (figure 3 ). LA132 with vinyl-vinyl backbones shows the lowest $\mathrm{T}_{\mathrm{g}}$ of $81.5^{\circ} \mathrm{C}$. This indicates that LA132 has the best flexibility to transfer the chain conformations when dispersing the cathode materials. With similar chemical composition, CMC and SA give similar DSC curves as well. Yet with the more rigid axial linkages, SA has a much higher $\mathrm{T}_{\mathrm{g}}\left(100.9^{\circ} \mathrm{C}\right)$ than $\mathrm{CMC}$ 's $\left(89.5^{\circ} \mathrm{C}\right)$, confirming the GAMESS calculation results mentioned.

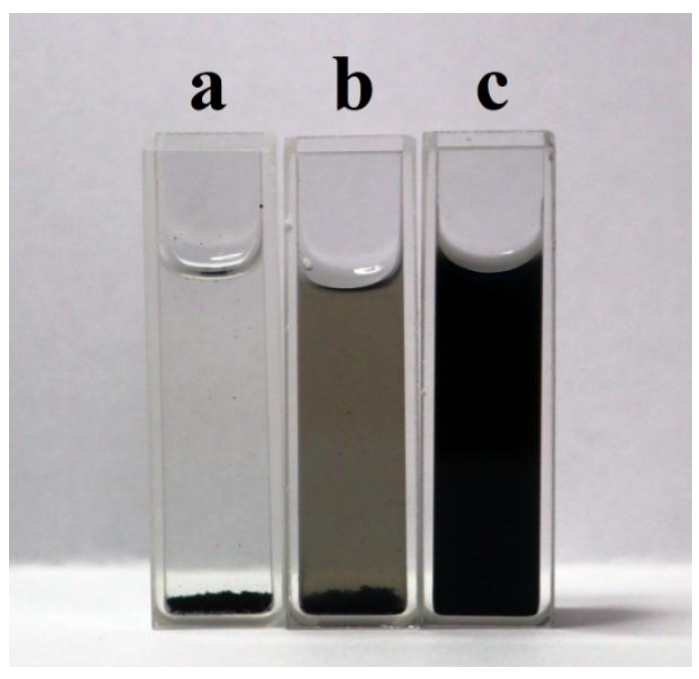

Figure 4. Suspensions of $1 \mathrm{mg} \mathrm{mL}^{-1}$ sulfur composites dispersed in (a) SA; (b) CMC; (c) LA132 after an overnight settlement.

With both factors of charge $(-\mathrm{COOH})$ density and chain flexibility having impacts, in order to illustrate the overall dispersability of the binders, the stability of the binder-S-KB colloids were examined. Three colloid samples of $1 \mathrm{mg} \mathrm{mL}-1$ 
S-KB particles dispersed in $1 \mathrm{mg} \mathrm{mL}-1$ of each binders, respectively, were prepared by ultrasonic treatment for 10 minutes.

The colloids obtained were then settled for overnight to allow the spontaneous coagulation. Figure 4 shows the suspensions after the settlement. The particles dispersed in SA solution experience severe aggregation and get completely coagulated. The coagulation in CMC solution is noticeable with some particles surviving. Compared to these, the particles in LA132 remain well dispersed, demonstrating the superior dispersability. The coagulation stems from the depletion effect[39, 40] between the particle and the binder chain. Depletion effect happens when the segmental adsorption energy of the polymer coil is insufficient to resist the loss of conformational entropy upon adsorption onto the particles[41, 42]. The depletion effect bring about a tendency of the chains to leave the particle surfaces. With less loss of conformational entropy, polymers with higher flexibility are suggested to better endure the depletion effect[43]. The stability of the cathode slurry would have dramatic effects on the morphology of the cathode. Upon drying, the binder chains tend to migrate with the removal of solvent, which facilitates the coagulation of the particles. Hence, the slurry with higher stability is able to better retain the well dispersed morphology of the wet state. In this way, LA132 shows the most optimal dispersability.

Additionally, the chain flexibilities not only effect the dispersability of the binders, but also influence the adhesion strength when the binders interact with the current collector substrate. Table 1 gives the peel test[44] results of the coated cathodes with the binders. The original shift-strain curves are given in the supplementary material Figure S1. When interacting with the Al substrate, the chains need to change their conformations to fit the rough surfaces of the substrate. As CMC is blended with SBR which is of even higher chain flexibility than LA132, the adhesive strength of CMC-SBR came higher than that of LA132. SA, as has been demonstrated to be rigid, displayed poor adhesive strength against peeling.

Table 1. Adhesive strength of the sulfur cathodes on the Al foil current collectors.

Binder SA
Adhesive strength $\mathbf{N} \mathbf{m m}^{-1}$ 
The specific discharge capacities $v s$. the cycle numbers for the cathode prepared by CMC-SBR, LA132 and SA,

respectively, are presented in Figure 5a. The results for the SA binder are in harmony with the dispersion performance. As the SA chains charge the S-KB and the SP particles least effectively, the aggregation in the pristine SA cathode leads to a lower initial discharge capacity $\left(990.8 \mathrm{mAh} \mathrm{g}^{-1}\right)$. Besides, owing to drastic coarsening, the capacity fading of the cathode with SA binder is significant as well (retention rate $67 \%$ after 50 cycle). LA132 achieves a better overall performance than CMC-SBR. Though CMC achieves better Zeta potential result, as LA132 resists the depletion effect better because of the better chain flexibility, the uniformity of the LA132 cathode is maintained during the drying process. This explains the comparatively lower initial discharge capacity of CMC-SBR cathode $\left(1046 \mathrm{mAh} \mathrm{g}^{-1}\right)$ than that of LA132 cathode (1169 $\left.\mathrm{mAh} \mathrm{g}^{-1}\right)$. However, the elastic SBR buffers the deformation caused by volume change during the cycling. Therefore, CMCSBR cathode achieves better capacity retention rate (78.5\% of the initial discharge capacity after 50 cycle) than LA132 (75.7\% of the initial discharge capacity after 50 cycle). The galvanostatic charge-discharge profiles of the cathodes with different binders at the $50^{\text {th }}$ cycle are given in Figure 5b. Compared to that of LA132 or CMC-SBR, the lower discharge plateau of SA indicates a greater polarization effect, which is probably because of the deformed electric conducting framework.
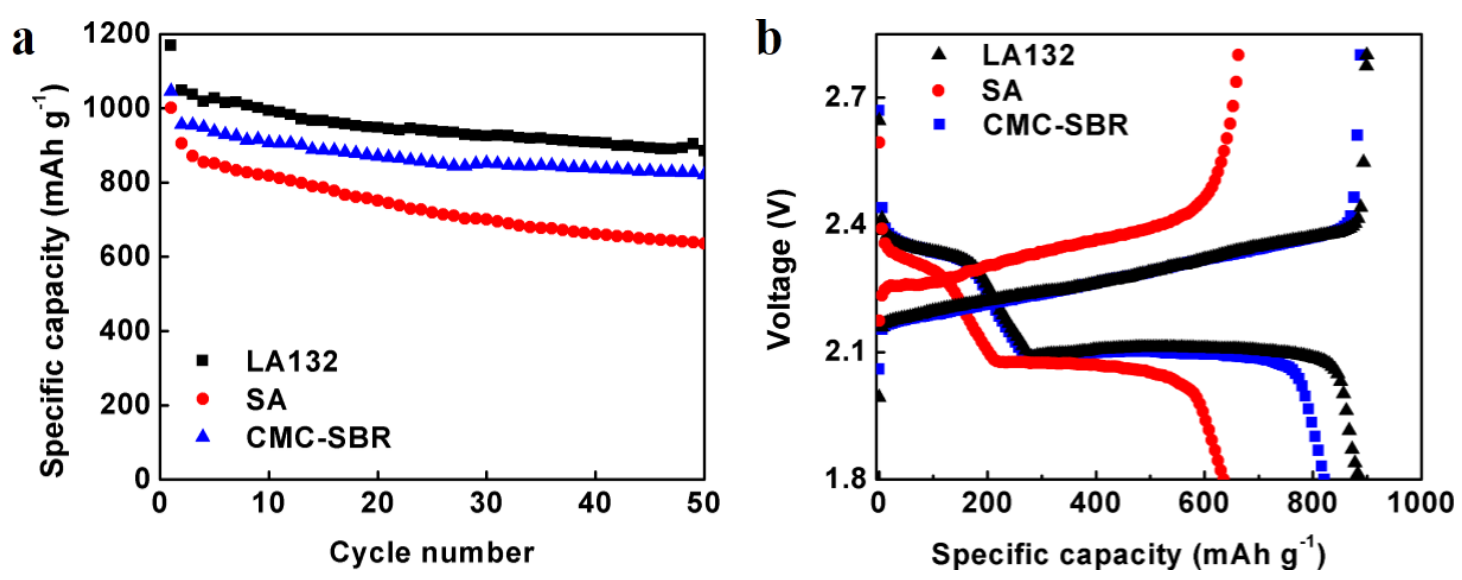

Figure 5. (a) Discharge capacity vs. cycle number of Li-S cells with different binders tested under galvanostatic conditions at $0.2 \mathrm{C}$; (b) the charge-discharge profiles of Li-S cells with different binders at the $50^{\text {th }}$ cycle tested under galvanostatic conditions at $0.2 \mathrm{C}$. 
To further look into how the binders effect the morphology of sulfur cathodes and the cell performances, the surfaces of the cathodes with different binders were observed before and after the cycling (figure 6). For the cathode with LA132 binder, as can be seen from figure $6 a$ and $b$, the cathode materials are finely dispersed at the pristine state and experience only slight coarsening, indicating both the dispersability and the structural stability of LA132. Besides, as mentioned, LA132 is a copolymer of acrylonitrile, acrylamide and acrylate where acrylonitrile makes up the basis. In aqueous environment, the hydrophobic $\mathrm{CH}_{2}-\mathrm{CH}-\mathrm{CN}$ segments would adsorb the alike hydrophobic surface of S-KB and SP particles. Meanwhile, the hydrophilic segments of acrylate and acrylamide segment would be solvated and wrap around the S-KB particles. The SEM image of micelles of LA132 formed in wet state is given in supplementary materials Fig. S2. This structure is able to cushion the volume expansion of the sulfur species and trap soluble polysulfide intermediates[8]. Compared to that of LA132 cathode, the morphology of the CMC cathode before (figure 6c) and after (figure 6d) the cycling are of higher granularity. The sulfur trapped inside the coarsened particles is less accessible for the $\mathrm{Li}^{+}$in the electrolyte. Besides, it is in less effective contact with the conducting framework as well. This leads to a lower utilization of electrochemical active sulfur and lower discharge capacity. However, as has been discussed, with the blending of elastic SBR which is noticeable from the rubbery structure in figure $6 c$, the cathode structure is imparted with better elastic robustness that enables higher capacity retention. For SA binder, owing to the poor dispersbility, the morphology of the pristine cathode (figure 6e) is much less uniform than that of CMC-SBR or LA132 cathode. As a result, the initial discharge capacity of the SA cathode $\left(990 \mathrm{mAh}^{-1}\right)$ is significantly lower. Without any buffering layers as LA132 offers or any elastic component as SBR, the SA cathode is not capable of enduring the deformation during the cycling. As shown in figure 6f, there are obvious cracks on the SA cathode, manifesting the vulnerability of the SA structure. Moreover, the distinct agglomeration of active sulfur on the surface of the cathode indicates severe polysulfide shuttling [1], which is in agreement with the cycling results. 

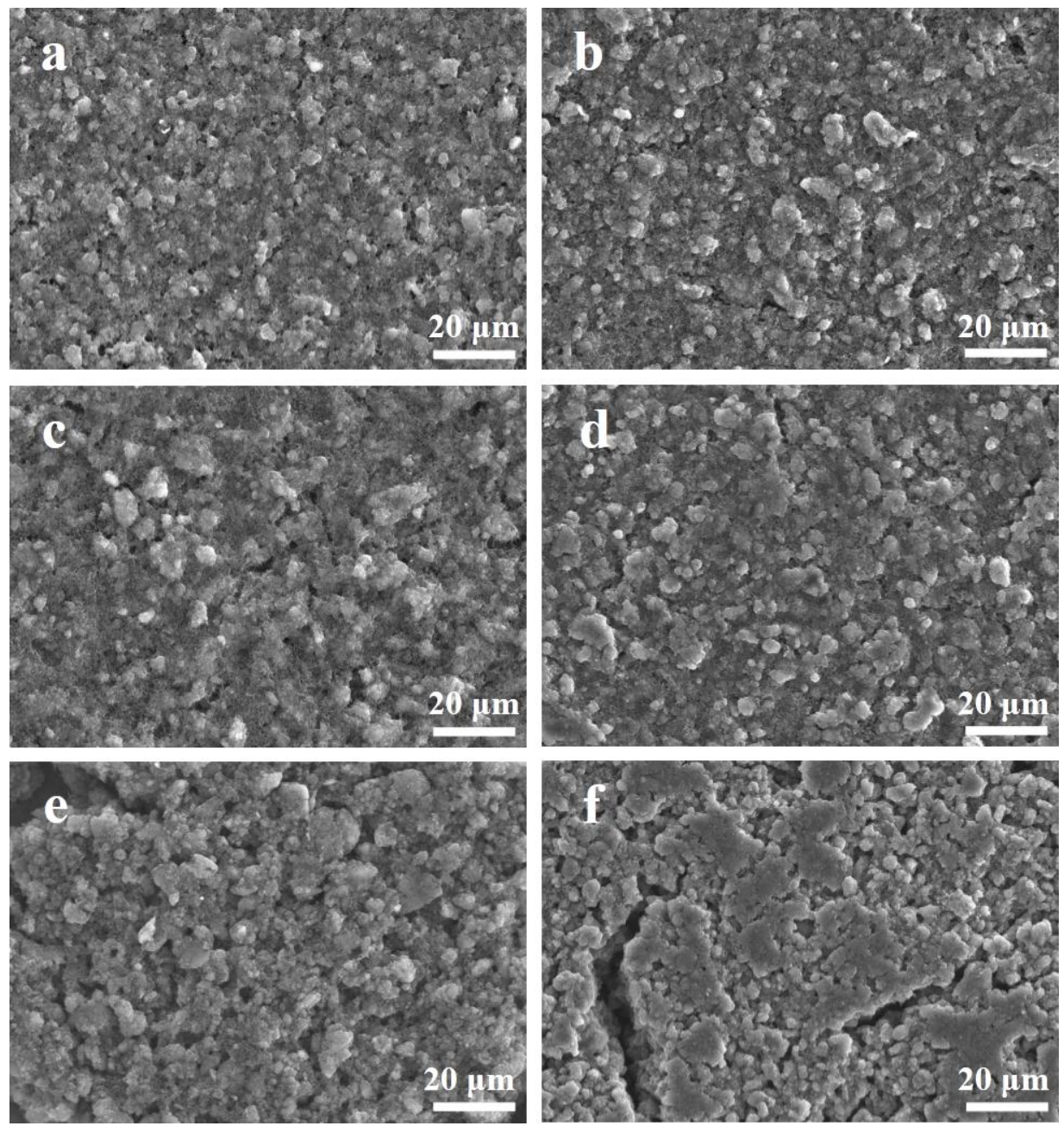

Figure 6. SEM images of the sulfur cathode (a) LA132 cathode before cycling, (b) LA132 cathode after 50 cycles, (c)

CMC-SBR cathode before cycling, (d) CMC-SBR cathode after 50 cycles, (e) SA cathode before cycling, (f) SA cathode after 50 cycles.

The outcomes are further confirmed by the EIS results. The Nyquist plots of the cells of different binders before and after the cycling are given in figure 7. The impedance curves of the cells before the cycling (figure 7a) appear as one depressed semicircle in high to medium frequency regions, followed by an inclined line in low frequency regions. The semicircles at the high to medium frequency regions reflect the charge transfer resistance $\left(\mathrm{R}_{\mathrm{ct}}\right)$ controlled by the electric conductivity of the cathode and the electrode-electrolyte interfacial resistance[45]. The higher $\mathrm{R}_{\mathrm{ct}}$ of SA cathode than that of LA132 or CMC-SBR cathodes implies the less effective electric contact within the cathode structure. As SA chains are restricted at changing conformations to charge the particle, the electric contact among the cathode materials was distributed less uniformly, resulting in the higher $\mathrm{R}_{\mathrm{ct}}$. Corresponding with the slightly higher granularity of CMC-SBR cathode, the $\mathrm{R}_{\mathrm{ct}}$ 
of the LA132 cathode is lower than that of the CMC-SBR cathode. The Nyquist plots of the cells after 50 cycles are given in figure $7 \mathrm{~b}$. The semicircles in the high frequency regions are attributed to the charge transfer resistance as well, while the semicircles in the medium frequency regions represent to the formation of $\mathrm{Li}_{2} \mathrm{~S}_{2} \mathrm{Li}_{2} \mathrm{~S}_{2}$ [45]. The $\mathrm{R}_{\mathrm{ct}}$ of the cathodes confirms the SEM results given in figure 6 that the LA132 better retains the cathode structure than CMC-SBR and the SA binder fails to resist the deformation of the cathode. What is worth mentioning is the similarity of the semicircles in the medium frequency regions, implying the formation of $\mathrm{Li}_{2} \mathrm{~S}_{2} \mathrm{Li}_{2} \mathrm{~S}_{2}$ in the cathode regardless of the binder species and the distribution morphology of the cathode materials. Hence, in order to suppress the formation of the $\mathrm{Li}_{2} \mathrm{~S} / \mathrm{Li}_{2} \mathrm{~S}_{2}$ layers, other methods e.g. chemical modification, structural design etc. are further required.
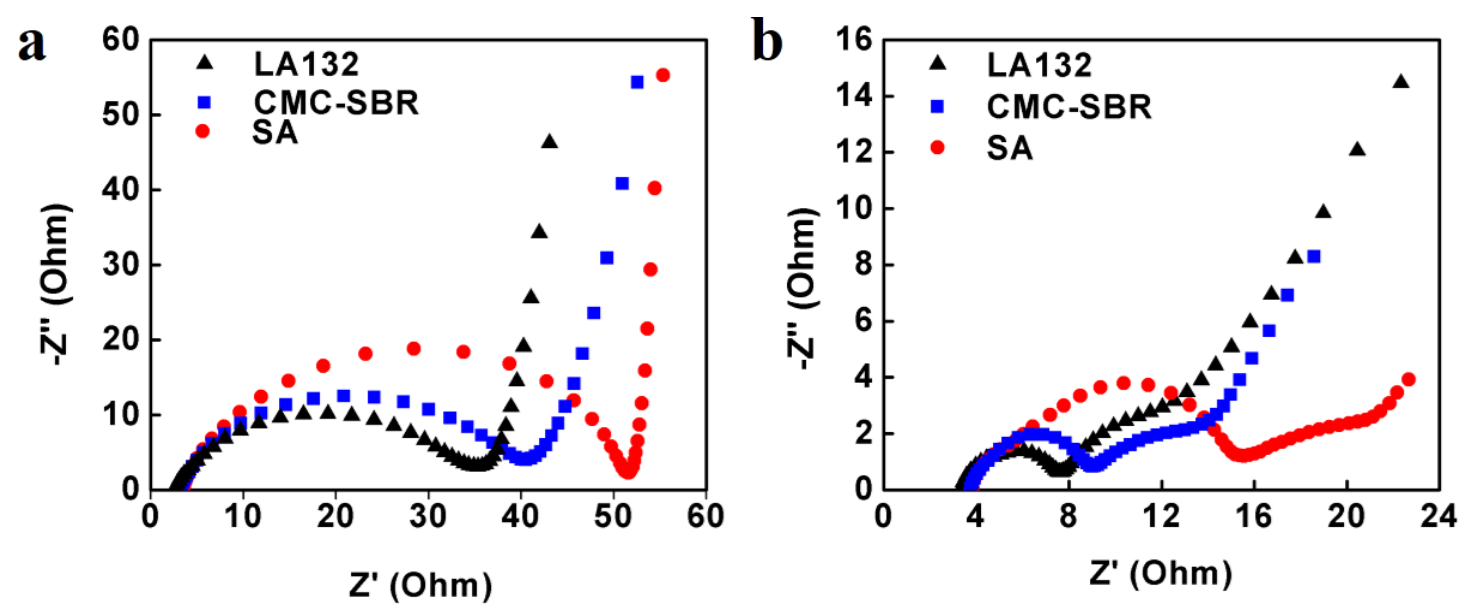

Figure 7. Nyquist plots of the cathodes with the binders in the frequency range of $10^{6} \mathrm{~Hz}$ to $0.1 \mathrm{~Hz}$. (a) before the cycling, (b) after the $50^{\text {th }}$ cycling

\section{Conclusions}

The impacting factors of the dispersability of commonly used ionic binders, LA132, CMC-SBR and SA, for Li-S were investigated in this study. The charge density, chain flexibility, electrostatic repulsion and depletion effect are found to have effects on the dispersion of cathode. To achieve cathode morphologies of higher uniformity, the binder is supposed to be of higher charge density and chain flexibility to enable favorable conformation that facilitates the electrostatic repulsion. Besides, higher chain flexibility is required for the binder to resist the depletion effect that break the adsorption. In spite of the similar chemical compositions of CMC and SA, the dispersability of CMC is much higher than SA, resulting from its higher chain flexibility stemming from the equatorial linkages with lower rotational barriers. With the vinyl-vinyl backbone 
structure of LA132 offering even higher chain flexibility, despite the lower anionic charge density distributed on the chains that decreased the zeta potential value, LA132 displays excellent dispersing stability compared to CMC or SA.

The electrochemical performances of the cells depend dramatically on the cathode morphologies influenced by the dispersbility of the binders. The cycling performance results agree well with the discussion about the dispersion of the binders. The cathode of LA132 reaches higher overall capacities over 50 cycles than that of CMC-SBR, and that of SA shows the lowest performances. The SEM images of the cathode morphologies further confirm that the cathodes with higher initial uniformities experience milder coarsening during the cycling and demonstrated higher overall electrochemical performances.

\section{Acknowledgements}

This work was financially supported by the National Science Foundation of China (NFSC) project No. 51373195 and No.51372263. Prof. B. V. R. Chowdari (Department of Physics, National University of Singapore) is greatly acknowledged for helpful discussion.

\section{Reference}

[1] X. Ji, L.F. Nazar, Advances in Li-S batteries, J. Mater. Chem., 20 (2010) 9821-9826.

[2] Y.V. Mikhaylik, J.R. Akridge, Polysulfide shuttle study in the Li/S battery system, J. Electrochem. Soc., 151 (2004) A1969-A1976.

[3] Y.X. Yin, S. Xin, Y.G. Guo, L.J. Wan, Lithium-sulfur batteries: Electrochemistry, materials, and prospects, Angew.

Chem. Int. Ed., 52 (2013) 13186-13200.

[4] X. Ji, K.T. Lee, L.F. Nazar, A highly ordered nanostructured carbon-sulphur cathode for lithium-sulphur batteries, Nature materials, 8 (2009) 500-506.

[5] N. Jayaprakash, J. Shen, S.S. Moganty, A. Corona, L.A. Archer, Porous Hollow Carbon@ Sulfur Composites for High - Power Lithium-Sulfur Batteries, Angew. Chem., 123 (2011) 6026-6030.

[6] J. Guo, Y. Xu, C. Wang, Sulfur-impregnated disordered carbon nanotubes cathode for lithium-sulfur batteries, Nano 
Lett., 11 (2011) 4288-4294.

[7] G. Zheng, Y. Yang, J.J. Cha, S.S. Hong, Y. Cui, Hollow carbon nanofiber-encapsulated sulfur cathodes for high specific capacity rechargeable lithium batteries, Nano Lett., 11 (2011) 4462-4467.

[8] H. Wang, Y. Yang, Y. Liang, J.T. Robinson, Y. Li, A. Jackson, Y. Cui, H. Dai, Graphene-wrapped sulfur particles as a rechargeable lithium-sulfur battery cathode material with high capacity and cycling stability, Nano Lett., 11 (2011) 26442647.

[9] Y. Cao, X. Li, I.A. Aksay, J. Lemmon, Z. Nie, Z. Yang, J. Liu, Sandwich-type functionalized graphene sheet-sulfur nanocomposite for rechargeable lithium batteries, PCCP, 13 (2011) 7660-7665.

[10] K.D. Ausman, R. Piner, O. Lourie, R.S. Ruoff, M. Korobov, Organic solvent dispersions of single-walled carbon nanotubes: toward solutions of pristine nanotubes, J. Phys. Chem. B, 104 (2000) 8911-8915.

[11] M.J. O'Connell, P. Boul, L.M. Ericson, C. Huffman, Y. Wang, E. Haroz, C. Kuper, J. Tour, K.D. Ausman, R.E. Smalley, Reversible water-solubilization of single-walled carbon nanotubes by polymer wrapping, Chem. Phys. Lett., 342 (2001) 265-271.

[12] S. Xin, L. Gu, N.-H. Zhao, Y.-X. Yin, L.-J. Zhou, Y.-G. Guo, L.-J. Wan, Smaller sulfur molecules promise better lithium-sulfur batteries, J. Am. Chem. Soc., 134 (2012) 18510-18513.

[13] D. Lv, J. Zheng, Q. Li, X. Xie, S. Ferrara, Z. Nie, L.B. Mehdi, N.D. Browning, J.G. Zhang, G.L. Graff, High Energy Density Lithium-Sulfur Batteries: Challenges of Thick Sulfur Cathodes, Adv. Energy Mater., (2015).

[14] S. Zhang, K. Xu, T. Jow, Evaluation on a water-based binder for the graphite anode of Li-ion batteries, J. Power Sources, 138 (2004) 226-231.

[15] Z. Cai, Y. Liang, W. Li, L. Xing, Y. Liao, Preparation and performances of LiFePO 4 cathode in aqueous solvent with polyacrylic acid as a binder, J. Power Sources, 189 (2009) 547-551.

[16] S.-E. Cheon, J.-H. Cho, K.-S. Ko, C.-W. Kwon, D.-R. Chang, H.-T. Kim, S.-W. Kim, Structural factors of sulfur cathodes with poly (ethylene oxide) binder for performance of rechargeable lithium sulfur batteries, J. Electrochem. Soc., 
149 (2002) A1437-A1441.

[17] M. He, L.-X. Yuan, W.-X. Zhang, X.-L. Hu, Y.-H. Huang, Enhanced cyclability for sulfur cathode achieved by a water-soluble binder, J. Phys. Chem. C, 115 (2011) 15703-15709.

[18] W. Bao, Z. Zhang, Y. Gan, X. Wang, J. Lia, Enhanced cyclability of sulfur cathodes in lithium-sulfur batteries with Na-alginate as a binder, J. Energ. Chem., 22 (2013) 790-794.

[19] M.J. Lacey, F. Jeschull, K. Edström, D. Brandell, Functional, water-soluble binders for improved capacity and stability of lithium-sulfur batteries, J. Power Sources, 264 (2014) 8-14.

[20] J. Wang, Z. Yao, C.W. Monroe, J. Yang, Y. Nuli, Carbonyl - $\beta$ - Cyclodextrin as a Novel Binder for Sulfur Composite Cathodes in Rechargeable Lithium Batteries, Adv. Funct. Mater., 23 (2013) 1194-1201.

[21] J. Sun, Y. Huang, W. Wang, Z. Yu, A. Wang, K. Yuan, Application of gelatin as a binder for the sulfur cathode in lithium-sulfur batteries, Electrochim. Acta, 53 (2008) 7084-7088.

[22] J. Pan, G. Xu, B. Ding, J. Han, H. Dou, X. Zhang, Enhanced electrochemical performance of sulfur cathodes with a water-soluble binder, RSC Adv., 5 (2015) 13709-13714.

[23] S.-R. Chen, Y.-P. Zhai, G.-L. Xu, Y.-X. Jiang, D.-Y. Zhao, J.-T. Li, L. Huang, S.-G. Sun, Ordered mesoporous carbon/sulfur nanocomposite of high performances as cathode for lithium-sulfur battery, Electrochim. Acta, 56 (2011) 9549-9555.

[24] M. Sun, S. Zhang, T. Jiang, L. Zhang, J. Yu, Nano-wire networks of sulfur-polypyrrole composite cathode materials for rechargeable lithium batteries, Electrochem. Commun., 10 (2008) 1819-1822.

[25] W. Zhou, Y. Yu, H. Chen, F.J. DiSalvo, H.c.D. Abruña, Yolk-shell structure of polyaniline-coated sulfur for lithiumsulfur batteries, J. Am. Chem. Soc., 135 (2013) 16736-16743.

[26] M. Rao, X. Song, E.J. Cairns, Nano-carbon/sulfur composite cathode materials with carbon nanofiber as electrical conductor for advanced secondary lithium/sulfur cells, J. Power Sources, 205 (2012) 474-478.

[27] M. Rao, W. Li, E.J. Cairns, Porous carbon-sulfur composite cathode for lithium/sulfur cells, Electrochem. Commun., 
$17(2012) 1-5$.

[28] J.-H. Lee, U. Paik, V.A. Hackley, Y.-M. Choi, Effect of carboxymethyl cellulose on aqueous processing of natural graphite negative electrodes and their electrochemical performance for lithium batteries, J. Electrochem. Soc., 152 (2005) A1763-A1769.

[29] B. Lestriez, S. Bahri, I. Sandu, L. Roué, D. Guyomard, On the binding mechanism of CMC in Si negative electrodes for Li-ion batteries, Electrochem. Commun., 9 (2007) 2801-2806.

[30] T.M. Riddick, Control of colloid stability through zeta potential, Blood, 10 (1968) 1.

[31] R.J. Hunter, Zeta potential in colloid science: principles and applications, first ed., Academic press, London, 1983. Charge and Potential Distribution at Interfaces.

[32] B. Medronho, A. Romano, M.G. Miguel, L. Stigsson, B. Lindman, Rationalizing cellulose (in) solubility: reviewing basic physicochemical aspects and role of hydrophobic interactions, Cellulose, 19 (2012) 581-587.

[33] Y. Qin, Alginate fibres: an overview of the production processes and applications in wound management, Polym. Int., 57 (2008) 171-180.

[34] K.Y. Lee, D.J. Mooney, Alginate: properties and biomedical applications, Prog. Polym. Sci., 37 (2012) 106-126.

[35] M.W. Schmidt, K.K. Baldridge, J.A. Boatz, S.T. Elbert, M.S. Gordon, J.H. Jensen, S. Koseki, N. Matsunaga, K.A. Nguyen, S.J. Su, T.L. Windus, M. Dupuis, J.A. Montgomery, General Atomic and Molecular Electronic-Structure System, J. Comput. Chem., 14 (1993) 1347-1363.

[36] G. Fletcher, M. Schmidt, M. Gordon, Developments in parallel electronic structure theory, Adv. Chem. Phys., 110 (1999) 267-294.

[37] J.-F. Su, Z. Huang, X.-Y. Yuan, X.-Y. Wang, M. Li, Structure and properties of carboxymethyl cellulose/soy protein isolate blend edible films crosslinked by Maillard reactions, Carbohydrate Polymers, 79 (2010) 145-153.

[38] R.J. Young, P.A. Lovell, Introduction to polymers, third ed., CRC press, New York, 2011. Chapter 16 :The amorphous state 
[39] A. Hanke, E. Eisenriegler, S. Dietrich, Polymer depletion effects near mesoscopic particles, Phys. Rev. E, 59 (1999) 6853.

[40] R. Hogg, T. Healy, D. Fuerstenau, Mutual coagulation of colloidal dispersions, Transactions of the Faraday Society, 62 (1966) 1638-1651.

[41] A. Milling, S. Biggs, Direct measurement of the depletion force using an atomic force microscope, Journal of colloid and interface science, 170 (1995) 604-606.

[42] A. Silberberg, Adsorption of flexible macromolecules. IV. Effect of solvent-solute interactions, solute concentration, and molecular weight, The Journal of Chemical Physics, 48 (1968) 2835-2851.

[43] D.H. Napper, Polymeric stabilization of colloidal dispersions, first ed., Academic Press, London, 1983. The effect of free polymer on colloid stability: theories of depletion flocculation and depletion phase separation.

[44] S. Komaba, N. Yabuuchi, T. Ozeki, Z.-J. Han, K. Shimomura, H. Yui, Y. Katayama, T. Miura, Comparative study of sodium polyacrylate and poly (vinylidene fluoride) as binders for high capacity $\mathrm{Si}$-graphite composite negative electrodes in Li-ion batteries, J. Phys. Chem. C, 116 (2011) 1380-1389.

[45] L. Yuan, X. Qiu, L. Chen, W. Zhu, New insight into the discharge process of sulfur cathode by electrochemical impedance spectroscopy, J. Power Sources, 189 (2009) 127-132. 